\title{
TUBERCULOUS PERICARDITIS WITH EFFUSION
}

\author{
BY \\ PHILIP ELLMAN \\ From the Leatherhead Emergency Hospital \\ Received May 28, 1945
}

This paper deals principally with effusive (rather than constrictive) tuberculous pericarditis, with special reference to a small series of cases that have come under my care since 1941 . There is a general belief that tuberculous involvement of the pericardium is very rare, at any rate in clinical practice. In view of the widespread distribution of pulmonary tuberculosis it would appear surprising that the pericardium should be so seldom attacked by the tubercle bacillus and it is of interest to ascertain whether this is, in fact, the case. My impression is that there has been a tendency to neglect tuberculous pericarditis, and to regard it as unduly rare, almost always fatal, and difficult to recognize in the living subject. I hope to show that, in fact, even acute forms of this disease may not necessarily be fatal and that healed tuberculous pericardial effusions may be a not uncommon cause of constrictive pericarditis.

Reported cases and personal communications from morbid anatomists with wide experience of tuberculosis suggest that a diagnosis of rheumatic or idiopathic pericardial effusion (or polyserositis) is made when, in fact, as with a neighbouring pleural effusion, the causative agent is really the tubercle bacillus. Holmes Sellors in his Hunterian lecture (1944) on constrictive pericarditis, not yet published, has emphasized the close relationship of tuberculosis and constrictive pericarditis.

Incidence. It has also been suggested that tuberculosis may follow hard upon rheumatic fever as a cause of pericarditis and that the condition is quite frequently overlooked clinically. Osler (1893) in 1000 autopsies reported 215 cases of tuberculosis in 7 of which (i.e. 0.7 per cent) tuberculous pericarditis was discovered. Norris (1911) in 7219 cases of tuberculosis from Philadelphia hospitals reported 1780 cases of tuberculosis of which 82 (1.1 per cent) had pericardial involvement. Harvey and Whitehill (1937) state that during 45 years 95 cases of tuberculous pericarditis were admitted to the Johns Hopkins Hospital: of these 71 were clinically important, 37 were proved tuberculous, and 34 were unproven, but these resembled in every respect the proven cases; it should, however, be noted that while the death rate of the proven cases was 83 per cent, of the unproven it was only 6 per cent. No other known cause, apart from tuberculosis, was established in the unproven cases. Cultures of the pericardial fluid were sterile but it is, of course, well known that tuberculous pleural effusions are not infrequently sterile on culture. Kornblum, Bellet, and Ostrum (1933) report an incidence in general autopsies of about 1 per cent, while in patients with pulmonary tuberculosis the autopsy incidence of pericardial involvement was 4 per cent. Gloyne informs me that in an analysis of 200 consecutive tuberculous autopsies which he made for Professor Lyle Cummins to compare with primitive tuberculosis in the Rand Native miners only one case was found in this series, as compared with 31 cases in the latter. Suzman (1944) analysed 1893 autopsies at Guy's Hospital and found that of the 102 tuberculous cases 6 (i.e. 6 per cent) had pericardial involvement.

Pathology. As regards the pathology we may recognize three main stages: (1) acute fibrinous (although commonly rheumatic); (2) effusive pericarditis with pericardial thickening; here if the amount of fluid is great there is extensive pressure on the surrounding structures, viz. the lungs, mediastinum, superior and inferior vena cava, mouths of the hepatic veins, together with dangerous pressure on the heart itself and great vessels within the pericardial sac (cardiac 
tamponade); large effusions without any appreciable cardiac enlargement are usually tuberculous, and the amounts may vary from 200-3000 c.c.; and (3) constrictive pericarditis (Pick's disease), the effusion having gradually subsided, leaving a greatly thickened pericardium (the effusions become smaller, thick and at times apparently, but not actually, purulent and then go on to fibrocaseous disease). If the condition is survived the adhesive process may progress to a complete obliteration of the sac with the eventual disappearance of the tubercles and caseation, the final result being an apparent non-specific chronic fibrous adhesive pericarditis. It can, therefore, be stated that in the absence of other causes constrictive pericarditis of a socalled non-specific type is probably tuberculous. Holmes Sellors has confirmed a tuberculous ætiology in most of the cases he has studied (i.e. 8 out of 9), and, as he points out, it may be deduced as axiomatic that rheumatic fever, including pericarditis, never produces the constrictive factor.

A primary tuberculous pericarditis may be regarded for all practical purposes as nonexistent. Tuberculosis may reach the pericardium (a) by the blood stream, as part of a miliary tuberculosis, or (b) by direct extension from a mediastinal focus. Study of reported cases and autopsy results suggest that the latter is the more likely and generally accepted means of secondary involvement of the pericardium, the infection taking place by extension along the lymphatic channels between the mediastinal glands and the pericardium. This was indisputably so in my own case of tuberculous pericardial effusion and pulmonary tuberculosis which came to autopsy, and in the autopsy report of a case of pericarditis by Barrett and Cole (1944). Direct extension from the lungs or pleura is almost unknown, which would explain the rarity of pericardial involvement without accompanying mediastinal gland involvement.

It seems probable, as Pagel (personal communication) has suggested, that most cases of tuberculous pericarditis occur in the course of an early dissemination following primary infection. The increase in late primary infection corresponds as he suggests with the contemporary rise in the incidence of tuberculous pericarditis in young adults. Cases occurring in the higher age groups and in senile tuberculosis are probably secondary to a tuberculous mediastinal gland involvement with a recrudescence of an early tuberculous process in the glands.

Symptomatology and physical signs of tuberculous pericarditis. These have been admirably described by many observers (Harvey and Whitehill, 1937, Hanneson, 1941, etc.) and I shall confine my attention purely to some points of particular value in clinical diagnosis. Unlike the rheumatic type of the disease the onset tends to be insidious rather than abrupt. There is seldom any considerable degree of local upset: lassitude, asthenia, and a slight evening temperature may be the principal general manifestations. Dyspnœa is not a pronounced characteristic in the cases reported, nor was it in my own cases, unless the effusion is a massive one, and in one such of my own series the patient would not admit to breathlessness and had been at work with this massive effusion right up to the time I saw her. Pain is present in varying degrees of intensity, bulging of the præcordium is noticeable, and there is frequently a cough (due to pressure on the trachea or bronchi), mostly non-productive, but if pulmonary tuberculosis is co-existent there is usually some sputum. In fact, as occurred in one of my own cases, the cardiac complications can be easily overlooked from a clinical standpoint. Often there is no œdema, but when it is present it frequently appears as a terminal event in massive effusions. With the more massive effusions, engorgement of the neck veins, invisible apex beat, diminished expansion, especially of the left hemithorax, together with a bulging of the epigastrium due to liver depression, as I shall describe later in Case 4, may be seen on inspection. Pericardial friction may sometimes be felt at the base of the heart and a palpable apex beat in the normal position may suddenly diminish or disappear as the effusion increases in size. Percussion may reveal a marked increase in the relative cardiac dullness to the right and leftdiagnostically this is of the utmost significance. Auscultatory phenomena of distant heart sounds and a friction rub, even on occasions with a large effusion (especially if adhesions are present), may be heard.

An outstanding feature of effusive or even constrictive pericarditis is the complete absence of significant murmurs, particularly noteworthy in view of the apparent size of the heart. 
The so-called Ewart's sign, associated with pulmonary compression with bronchial breathing and whispering pectoriloquy at the angle of the left scapula, is sometimes heard. It was present in two of my cases.

A low arterial and pulse pressure and high venous pressure with a quietly acting heart and a paradoxical pulse (radial pulse almost disappearing on deep inspiration) may occur, especially with massive effusions and those going on to the stage of constrictive pericarditis. The socalled Beck's triad in acute and chronic compression is noteworthy. In acute cardiac compression (tamponade) with massive pericardial effusion Beck describes (1) a low arterial pressure, (2) a high venous pressure, and (3) a quiet heart, although Volhard refers more accurately to a small heart. In chronic cardiac compression (constrictive pericarditis) there is (1) ascites and a large liver, (2) high venous pressure, and (3) a quiet heart. This syndrome must not be confused with a polyserositis, i.e. Concato's disease, but it may be consequent upon it (Paul White), as in my own fourth case. Thus, as emphasized by White (1935), Hanneson (1941), and Sellors (1944), polyserositis as a cause rather than effect of constrictive pericarditis is well recognized, but the conditions may merge imperceptibly into each other. With the development of constrictive pericarditis the low arterial pressure becomes associated with a pulse pressure as low perhaps as 20 .

$X$-ray examination of the heart. This is often the only reliable means of early diagnosis and from this point of view we owe much to the comprehensive study of Roesler, to which those interested are referred. With moderate-sized effusions the cardiac silhouette presents a pear-shaped outline in the erect position, the shape changing in the recumbent position to a globular or rectangular appearance. This change in shape with change in position may be of considerable diagnostic significance as will be shown from a description of one of my cases. Fluoroscopy further assists in the diagnosis from gross cardiac enlargement by demonstrating the presence of diminished or even absent cardiac pulsation, both in massive effusive pericarditis and in constrictive pericarditis. Kymography, when available, gives a valuable permanent record of these fluoroscopic findings and Sellors reported it to be of considerable diagnostic value in one of his cases.

Electrocardiographic examination may, as a general rule, be of diagnostic value, often closely resembling that of an acute myocardial infarction with characteristic changes in the $\mathrm{R}-\mathrm{T}$ segments, in the $\mathrm{T}$ waves, and with the low voltage curves in all leads. Unlike myocardial infarction, however, the $\mathrm{Q}$ wave is not exaggerated and the direction of the $\mathrm{R}-\mathrm{T}$ segment and the $T$ waves are the same in all leads.

Diagnostic pericardial puncture. The one infallible diagnostic procedure is the recovery of pericardial fluid by paracentesis, and this can be safely adopted where symptoms, signs, and $\mathrm{X}$-ray examination indicate it. The correct diagnosis is conclusively determined in pericardial as in pleural effusions - and with equal facility - by pericardial puncture. Pericardial paracentesis is always advisable both for diagnostic purposes and to relieve pressure; if excessive some $300-400$ c.c. or more may be removed and replaced by half the quantity of air (gas replacement).

The site usually selected is the fifth left interspace outside the apex beat if this is palpable and just over $1 \mathrm{~cm}$. within the outer border of cardiac dullness. Preliminary adequate anæsthestization with 2 per cent novocaine of the skin subcutaneous tissue right up to the pericardium is essential for success, exactly as it is in the case of pleural effusions. Occasionally, where examination shows more fluid to the right or posteriorly at the angle of the left scapula, a site at the fourth right interspace or posteriorly at the angle of the left scapula may be chosen. A 20 or 50 c.c. two- or three-way syringe is valuable if it is proposed to induce a pneumopericardium, and the type of needle used for pleural punctures is adequate as a general rule, unless the fluid is of a thick consistency and the pericardium is thickened, when a widebored needle may be essential.

Induction of an artificial pneumopericardium. This measure was first reported by Wenckebach in 1910, and is particularly indicated for large effusions that require repeated tapping on account of rapid re-accumulation of fluid. The fluid is usually replaced by half the quantity of filtered air. Apart from the symptomatic relief my impression that the re-accumulation of fluid is thereby retarded is reinforced by the course of events in one of my own cases. The 
chief therapeutic advantages of this procedure may be regarded as the prevention of rapid re-accumulation of the fluid, the prevention of the formation of adhesions by keeping the pericardium distended with air although this is a doubtful sequence, and the demonstration of the size of the heart which is found to be invariably enlarged with a rheumatic lesion and normal or only moderately enlarged with a tuberculous lesion (see Fig. 7B).

Prognosis. The prognosis of pericardial effusions is naturally dependent upon the nature of the effusion, i.e. whether it is clear, hæmorrhagic, or purulent. Most massive clear effusions and some hæmorrhagic ones are tuberculous.

The mortality rate has hitherto been regarded as very high but recent experiences are certainly suggesting modification of this view. In the same way that it is often difficult to recover the tubercle bacillus from clear pleural lymphocytic exudates, which are sterile on culture (now almost universally recognized as tuberculous in young subjects) so with pericardial exudates similar difficulties present themselves. The general impression is that if tubercle bacilli are readily obtained from the pericardial fluid the prognosis is a grave one (Harvey and Whitehill, 1937). In my fatal case the pericardial fluid abounded with tubercle bacilli. Even guinea-pig inoculations are not always successful in isolating the tubercle bacillus, e.g. Suzman (1944) reported a case of tuberculous pericardial effusion, proved at autopsy, where, during life, the fluid was repeatedly sterile on culture, the Mantoux negative, and a guinea-pig test negative on two occasions. Sellors (1944), in a young girl with a massive tuberculous pericardial effusion found on paracentesis that the fluid was negative for tubercle bacilli. Culture and guinea-pig inoculation were likewise negative yet at operation for pericardial resection a few weeks later the pericardium-of the bread and butter type-was found to be riddled with tuberculous lesions.

Barrett and Cole (1944) report a case where the fluid was sterile on culture and where, when it was injected into a guinea-pig the animal maintained health for the unusually long period of five months. Subsequently it died, exhibiting tuberculosis.

In two unproven cases from my own series the fluids injected into guinea-pigs (killed three months after inoculation) were negative but in every way characteristically tuberculous. As far back as 1917, Parkinson (personal communication) reporied a case of proved tuberculous pericardial effusion, which I shall quote in greater detail later, where repeated examinations of the pericardial fluid were sterile on culture.

It is therefore obvious that, from the pathological point of view, a negative fluid by no means excludes tuberculosis. On the contrary, where, in spite of a negative fluid, everything else points to the diagnosis of a tuberculous lesion, my impression has been that the prognosis may be regarded as correspondingly more favourable.

Treatment. As with pleural effusions the general constitutional aspect of the case is important. Once the diagnosis has been made in hospital the aim should be a careful sanatorium regime with its strict routine and discipline. From the point of view of local treatment mercurial diuresis may be considered, particularly in polyserositis, while pericardial paracentesis should be repeated where there is a massive effusion. The value of taking the venous pressure and doing an air replacement (pneumopericardium), even if only to determine the size of the heart and the thickness of the pericardium, should be appreciated. Where Beck's triad with chronic cardiac compression originated from a pericardial effusion or a polyserositis (provided that the tuberculous process is reasonably quiescent) surgery, in the form of pericardial resection, should be given serious consideration in view of some of the brilliant results that have been reported (Paul White 1935, Holmes Sellors 1944).

The following case reports will serve as an illustration of some of the points already mentioned.

\section{Case 1.}

\section{CASE Notes}

A factory worker, aged 22, seen in January 1944, gave the following history: three weeks before Christmas she had an attack of " influenza " when she complained of headaches, giddiness, nausea, and tiredness in the legs. These symptoms lasted two days, and a week later she noticed slight swelling of the ankles and dyspnœa. She had no chest pain, palpitation, nor appreciable loss of weight. 

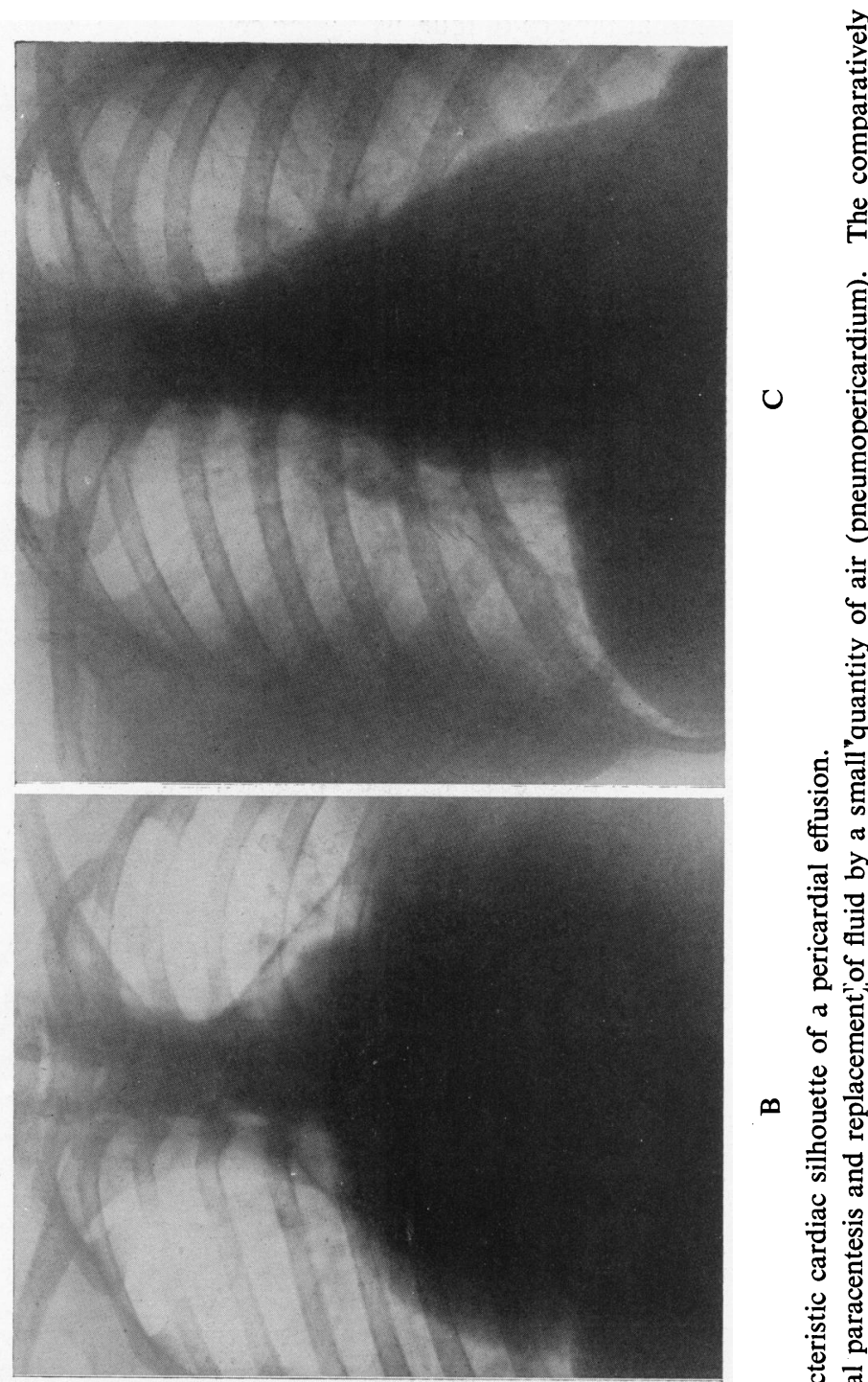

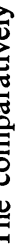

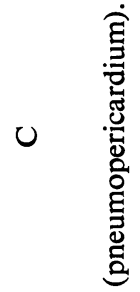

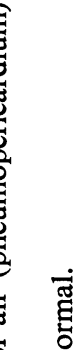

虫

密 壱

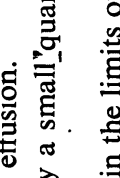

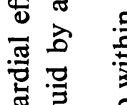

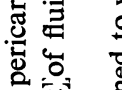

范 吾

๑

芯 흘 무

\%

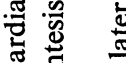

¿ \&

㚬

品

苋哥客

究需

哩需

里

㖓

을

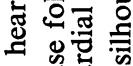

\& छ .

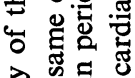

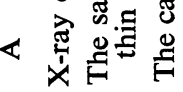

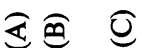

ت. 
The past history included measles at the age of four, whooping cough at five, and scarlet fever at seven; there was no history of chorea or rheumatic fever and no family history of tuberculosis. On admission to Leatherhead Emergency Hospital she was obviously ill, pale, and toxic. There was slight cyanosis of the lips, some dyspnœa, and some engorgement of the neck veins, but the girl was able to lie flat. Temperature range 97-100; pulse regular and 120; respirations 24. Mantoux test, 1 in 1000 , was positive.

Examination of the heart. Apex beat not palpable; heart greatly enlarged to right and left; area of cardiac dullness $8 \mathrm{~cm}$. to right of sternum and $17 \mathrm{~cm}$. to left; heart sounds faint; rhythm regular; blood pressure 135/100 ; no murmurs heard; no friction audible; signs of compression at the left base.

Fig. 1A shows gross enlargement of the cardiac silhouette, characteristic of a pericardial effusion.

A cardiogram showed low voltage curves and flat $T$ waves in all leads, quite in keeping with pericardial effusion. The $T$ waves were improved in a subsequent.cardiogram.

Blood count: red cells 6.5 million; hæmoglobin 56 per cent; colour index 0.4 ; white blood cells 8500; nothing abnormal in differential count.

Pericardial paracentesis yielded 600 c.c. of clear, straw-coloured fluid which clotted readily on standing: S.G. 1030; total protein 4.7 g. per 100 c.c.; albumin 3.9; globulin 0.8; culture sterile. A pneumopericardium was attempted but she felt faint after 100 c.c. of air had been introduced and it was discontinued (Fig. 1B).

Guinea-pig inoculation: animal killed two months later, no evidence of tuberculosis.

Repeated pericardial paracentesis, performed at intervals, yielded only small quantities of serosanguinous fluid; blood sedimentation rate was 10 .

Two weeks later the cardiac dullness had become greatly reduced but pericardial friction now heard to the left of the sternum. In the third month her condition had improved enormously: her pulse rate became normal. An X-ray showed a cardiac silhouette approaching the normal (Fig. 1C). The cardiogram showed better formation of waves T I and T II. On discharge hæmoglobin was 98 per cent.

Seen at regular intervals since her discharge, during which time she has followed a careful sanatorium regime, this girl has no clinical or X-ray evidence of cardiac abnormality and is back at work. I have little doubt that her condition was due to a tuberculous pericardial effusion.

\section{Case 2.}

A married woman, aged 34, admitted to the Leatherhead Emergency Hospital complaining of substernal tightness and pain, palpitation, lassitude, loss of weight, and slight breathlessness of three months' duration.

Her previous history was a good one with no evidence of rheumatism. Her mother had died of pulmonary tuberculosis.
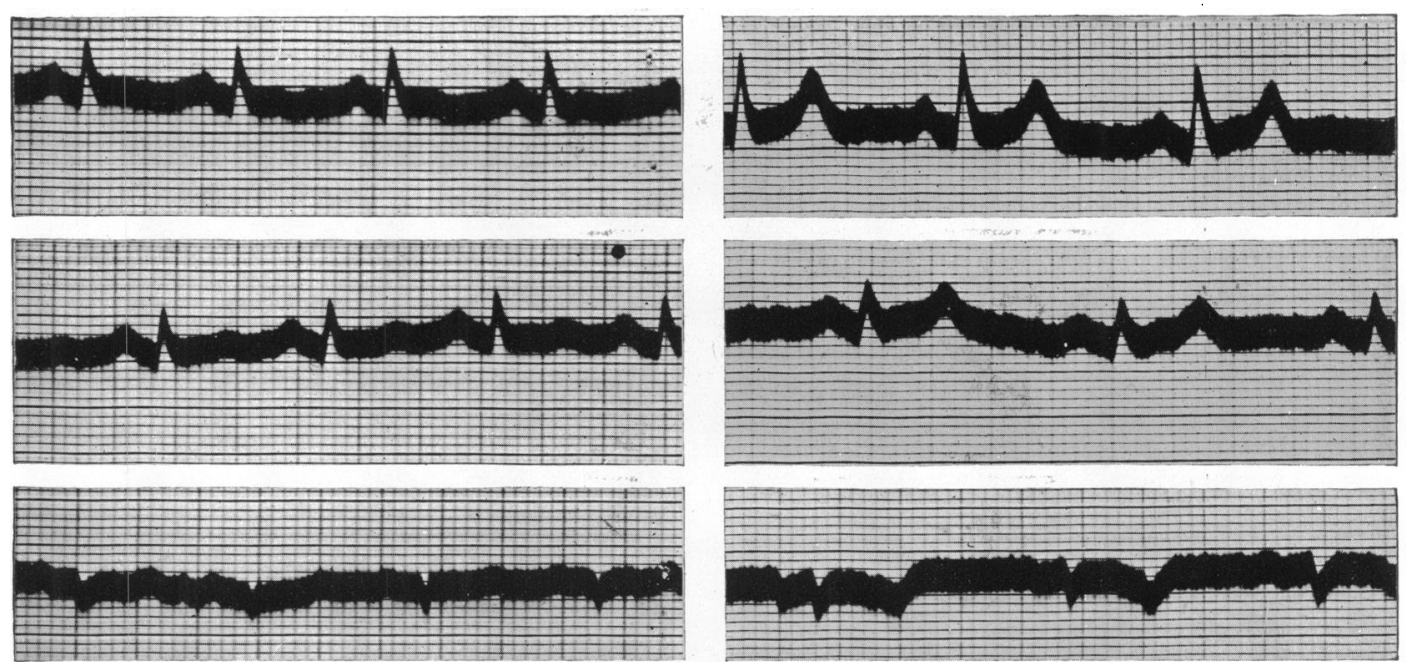

A

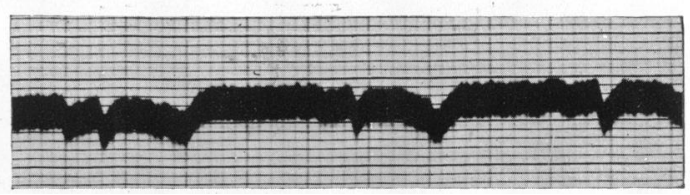

B

FIG. 2.-Case 2. (A) Electrocardiogram before treatment: showing low voltage deflections and slight left axis deviation. (B) After treatment: $R$ is now higher voltage and T I and T II are considerably improved. 
She was not cyanosed; temperature intermittent (97-99.4); pulse 90 and regular; respirations averaged 24; no engorgement of neck veins; blood sedimentation rate 39 ; blood count normal.

Examination of the heart. Apex beat in normal position, but an increased area of cardiac dullness to left and right; no murmurs; pericardial friction heard at base; B.P. 120/90. X-ray examination ; confirmed the clinical diagnosis of pericardial effusion.

A cardiogram showed small low voltage deflections and flat $\mathrm{T}$ waves in lead I. Following treatment subsequent records showed higher voltage curves and improvement in $\mathrm{T}$ waves (Fig. 2A and $\mathrm{B}$ ).

Pericardial paracentesis on two occasions yielded a blood-stained effusion which, on examination, showed a moderate number of leucocytes ( 82 per cent lymphocytes); total protein 5.8 g. per 100 c.c.; direct smear showed no organisms; culture sterile; guinea-pig inoculation negative after three months.

This case is regarded as a case of tuberculous pericardial effusion in spite of the negative guinea-pig test. Serial clinical, radiological (Fig. 3A and B), and cardiographic examinations have, since her dis-

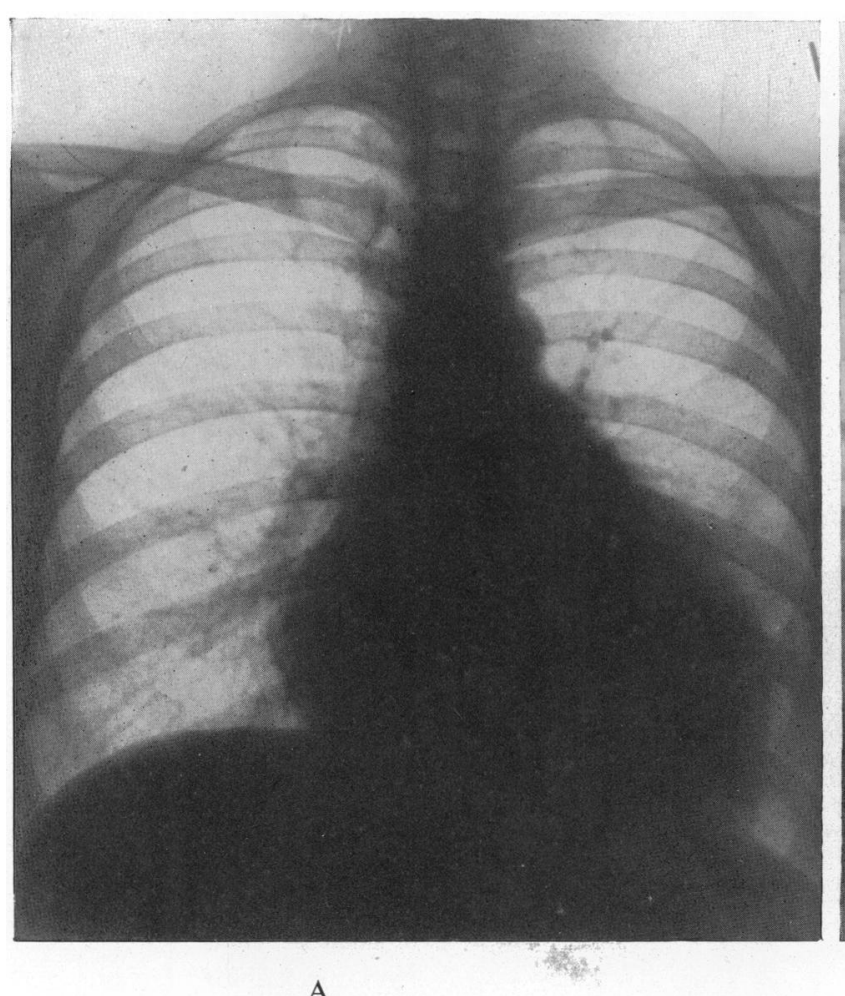

A

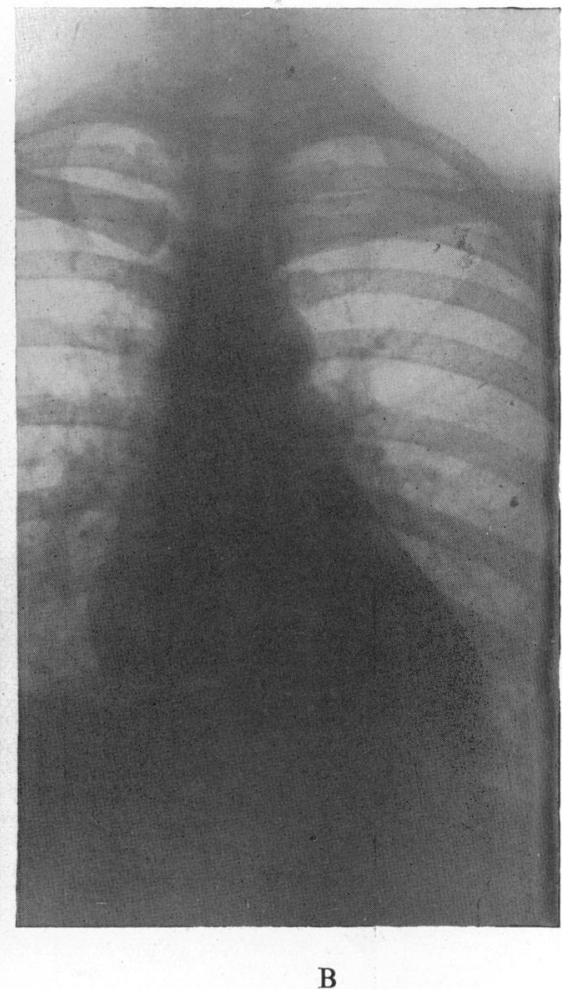

FIG. 3.-Case 2. (A) Characteristic cardiac silhouette of a pericardial effusion. (B) Showing cardiac silhouette almost within the limits of the normal, following pericardial paracentesis.

charge nearly three years ago, shown progressive improvement. She now has no symptoms nor any cardiac abnormality.

\section{Case 3.}

A labourer, aged 52, had extensive bilateral pulmonary tuberculosis and tubercle bacilli were present in large numbers in the sputum. He was in a sanatorium for six months in 1942, where he made satisfactory progress and gained 1 stone in weight, but the sputum remained bacilliferous. There was then no evidence of any cardiac disease (Fig. 4A).

When seen again a year later he was much worse, was cyanosed, and had a cough with much sputum. Temperature 101; pulse 130; and clinical examination showed obvious re-activation of the disease in both lungs. It was, however, with the greatest astonishment that I discovered on the routine skiagram, in addition to the extensive lung infiltration with cavitation, a cardiac silhouette highly suggestive of a pericardial effusion (Fig. 4B). The patient died in the sanatorium four days later.

Post-mortem examination (confined to the chest). Both pleuræ obliterated by fibrous adhesions; massive caseous glands in both hilar regions. Lungs showed extensive fibrocavernous disease. Pericardial cavity appeared to occupy nearly two-thirds of the thoracic cavity and contained a thick, blood-stained, loculated effusion. 


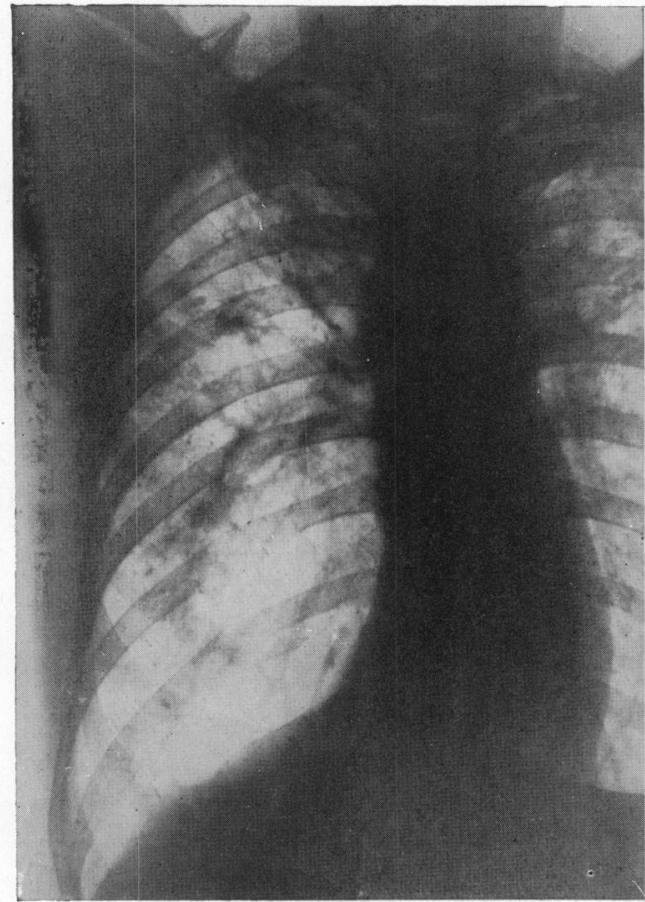

A

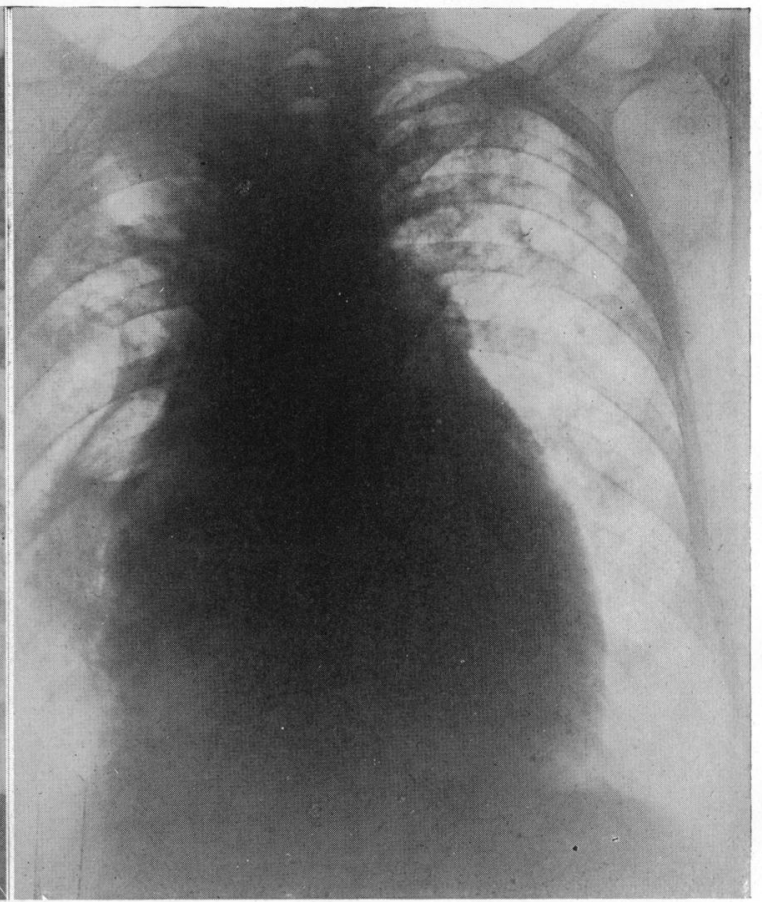

B

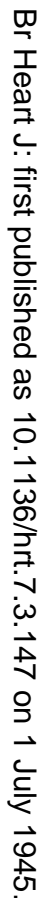

Fig. 4.-Case 3. (A) X-ray of the chest, showing extensive lung infiltration with cavitation, inyolving upper and middle zones of both lungs. (B) The same case twelve months later, showing extensive lung infiltration with cavitation complicated by a massive pericardial effusion.

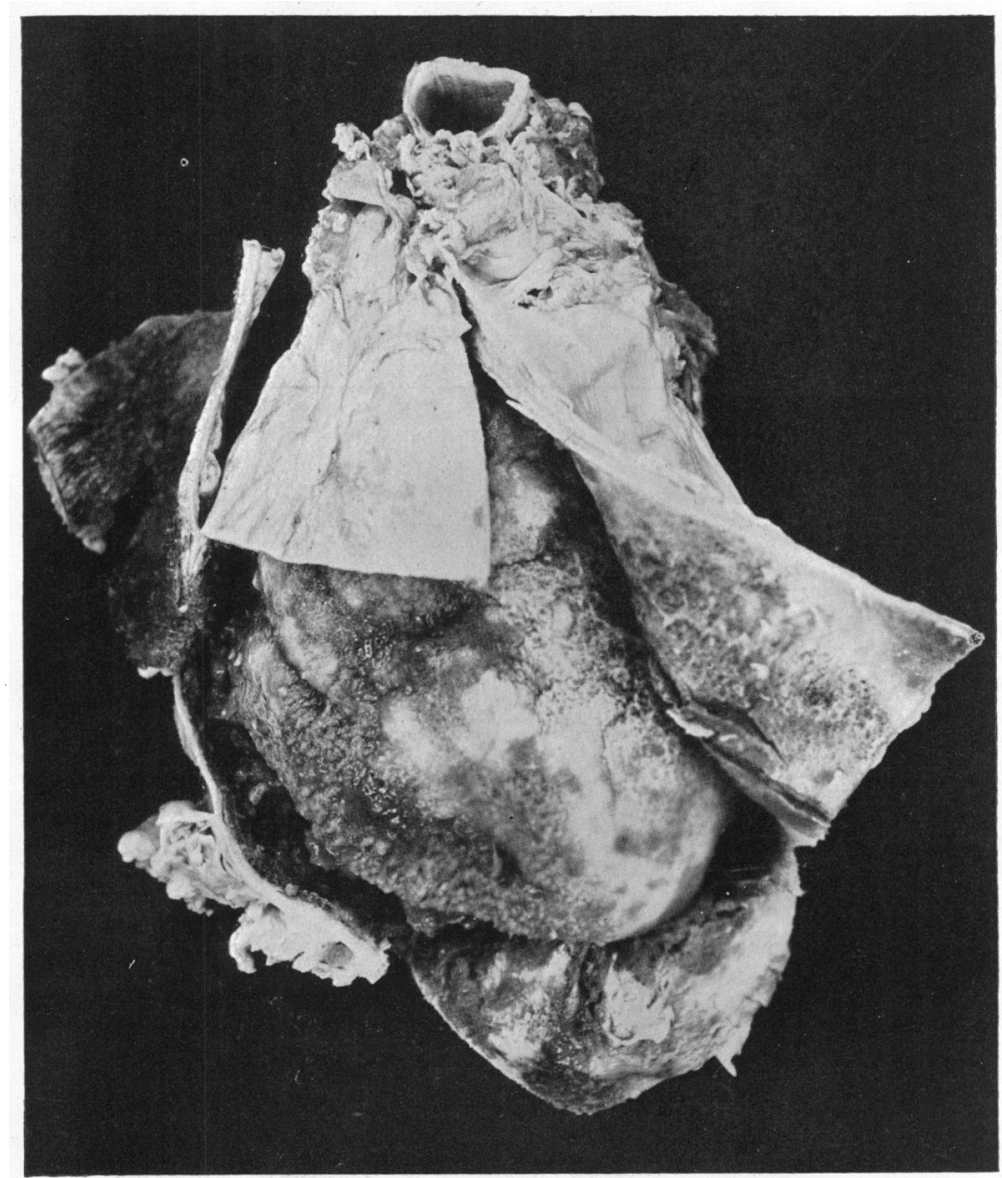

con 
Dr. J. N. Cummings, who examined the heart and pericardium very completely, also kindly photographed it for me and took sections of the pericardium of which he has been good enough to supply me with microphotographs. He reports as follows on the heart, pericardium, and pericardial fluid.

The heart was considerably enlarged, measuring $14.5 \times 10.7 \mathrm{~cm}$. after removal of pericardial sac.

Left ventricle hypertrophied, thickness of left ventricle wall being just under $2.5 \mathrm{~cm}$. from apex. Muscle covered all over by a thick visceral pericardium which varied from 0.5 to $1 \mathrm{~cm}$. and was composed of a granular pink- or grey-coloured granulation tissue. Parietal pericardium grossly thickened, being 0.5 to $1 \mathrm{~cm}$. in thickness and consisting (naked eye) of fibrous tissue and granulation tissue with considerable irregularity of surface. On the irregular surface of the parietal pericardium in the fixed specimen were considerable collections of rather gelatinous material, some up to $1 \mathrm{~cm}$. in thickness (Fig. 5).

Histological examination. Sections were cut of the pericardium, and also of the heart muscle. No tuberculous lesions were found in the heart muscle itself. The section of the pericardium showed a fibrinous exudate lying upon typical tuberculous granulation tissue. Giant cells and tubercle formation could be found with ease. There was a fair amount of mononuclear cellular infiltration throughout. Tubercle bacilli found in some numbers in the section (Fig. 6).

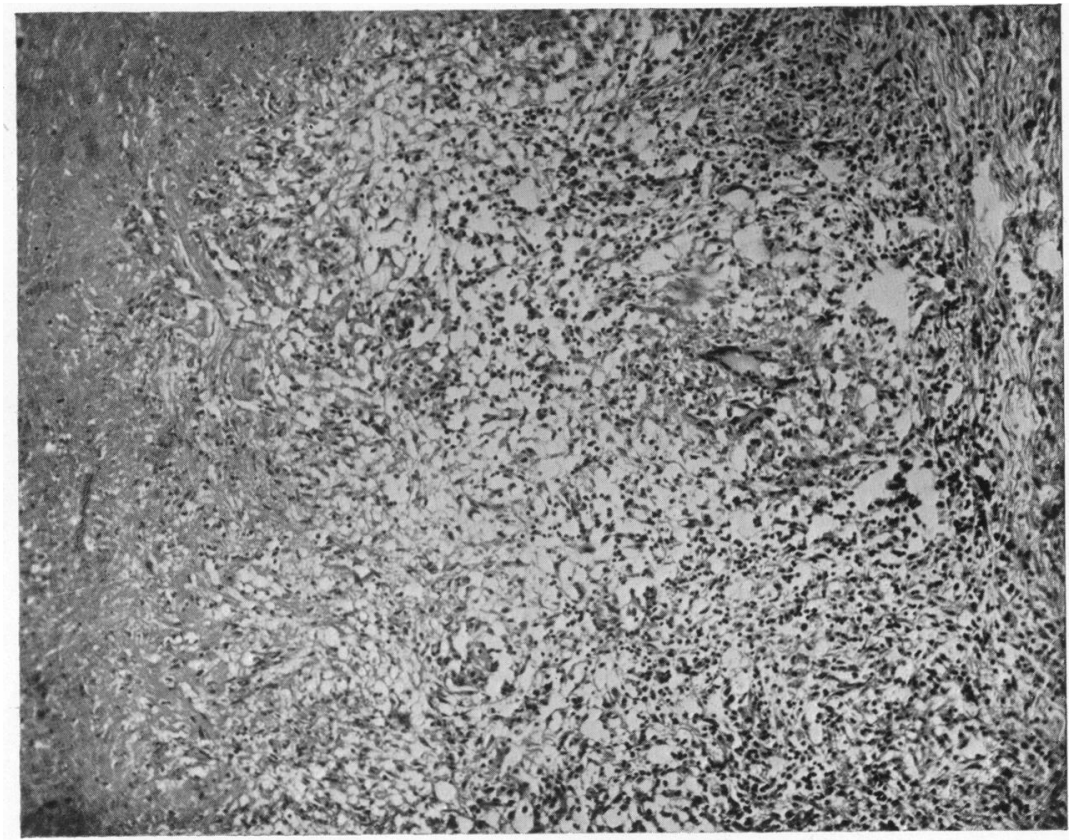

FIG. 6.-Case 3. Microphotograph of the pericardium, showing typical tuberculous granulation tissue and giant cell formation. Magnification $\times 50$.

Pericardial fluid: blood-stained; red blood cells 47,000 per cub. mm.; white cells 900 per cub. mm.; polymorphs 10 per cent; lymphocytes 90 per cent; protein 3.0 per cent; specific gravity 1040; direct smears, a very few tubercle bacilli present; cultures, a fair growth in two weeks.

\section{Case 4.}

A gardener, aged 20, had an acute attack of "influenza" in February 1941, after which he complained of increasingly severe breathlessness, a cough with a trace of mucoid sputum, and some swelling of the legs. His previous history showed no noteworthy illness, nor was there anything relevant in the family history.

Admitted to hospital seven weeks after onset of illness, very ill and grossly orthopnœic; temperature range 97 to 102.8; pulse 130; no appreciable chest pain; no cyanosis or engorgement of the veins of the neck.

Examination. The chest showed marked limitation of movement on both sides, but especially the left; bulging of the præcordium; marked dullness over the left side up to the second intercostal space; breath sounds absent over areas of dullness.

Heart: apex beat not then palpable; heart sounds distant and feeble; rhythm regular; rate 130; B.P. 130/75; no significant murmurs, but for three weeks after admission pericardial friction heard at base of heart. 
Abdomen: ascites; liver palpable; some œdema of legs; urinary output at first diminished but became normal.

X-ray examination of chest showed a bilateral pleural effusion, particularly marked on left. After aspiration of left-sided effusion cardiac silhouette markedly increased and strongly suggestive of pericardial effusion (Fig. 7A).

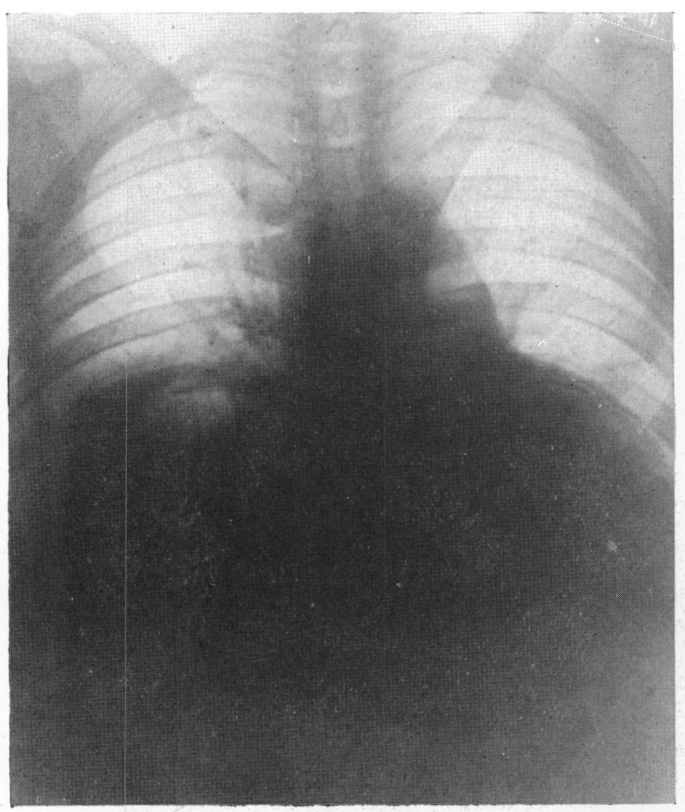

A

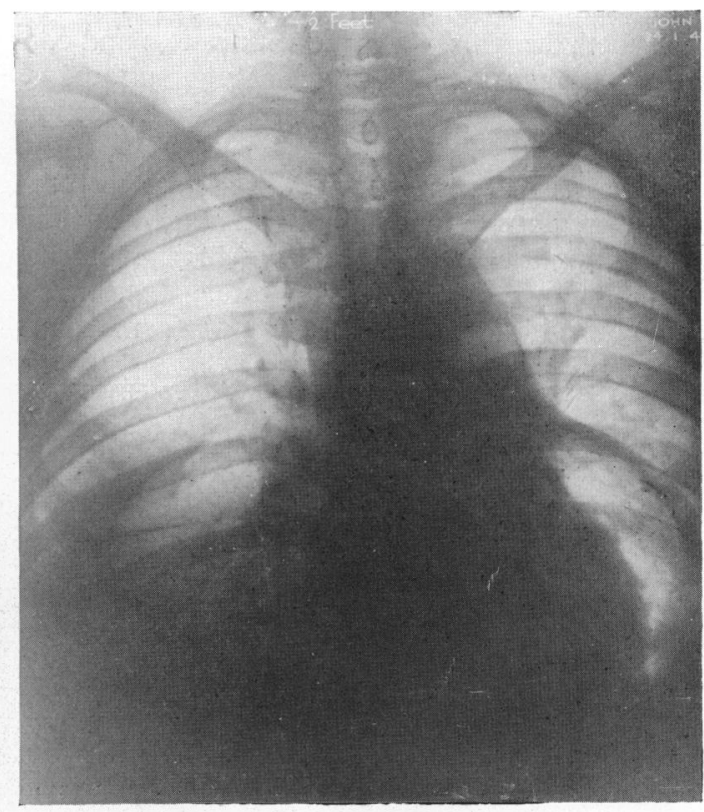

B

Fig. 7.-Case 4. (A) The left pleural effusion has been aspirated; the right pleural effusion and the pericardial effusion are well seen. (B) Following pericardial paracentesis and replacement by air: the size of the heart and the thick pericardium are well seen.

Electrocardiogram showed rather low voltage curves in limb leads; $T$ waves in all leads negative, especially præcordial.

All these findings were highly indicative of an acute polyserositis, including a big pericardial effusion, which was confirmed by paracentesis of pleuræ and later of pericardium.

Biochemical and pathological investigations: $21 / 4 / 41$.

Pleural fluid: clear, straw-coloured, some clotting; polymorphs 2 per cent; lymphocytes 98 per cent; culture sterile; Ziehl-Neelsen, no tubercle bacilli seen; total protein $4.65 \mathrm{mg}$. per 100 c.c.; serum albumin 2.06; serum globulin 2.59; albumin globulin ratio 0.79 .

Pericardial fluid: clear, straw-coloured fluid; polymorphs 90 per cent; lymphocytes 10 per cent; culture sterile; Ziehl-Neelsen, no tubercle bacilli seen; total protein $4.7 \mathrm{mg}$. per 100 c.c.; serum albumin 1.75; serum globulin 2.95; albulmin globulin ratio 0.59 .

The differences between the cytology of the pleural and pericardial fluids are noteworthy.

Guinea-pig inoculation with pleural and pericardial fluids both showed tubercle in spleen and lungs.

Urine: albumin, a trace; deposit; an occasional red and white blood cell seen; direct smear, no organisms seen; culture, no growth.

Blood: B.S.R., $34 \mathrm{~mm}$. at end of one hour; hæmoglobin, 78 per cent.

\section{Treatment and progress.}

The patient was treated on general lines for his tuberculosis and paracentesis of pleuræ and pericardium were performed. The fluid in the pericardium was replaced by air on several occasions and there was clinical evidence of a hydro-pneumopericardium with characteristic auscultatory physical signs of a " tinkling splash" over the præcordium. He also had a course of salyrgan.

An X-ray film in January 1942 (Fig. 7B) showed a pure pneumopericardium with no fluid and a corresponding disappearance of auscultatory signs. The thickened pericardium was well seen radiologically and the heart did not appear enlarged. Ascites and oedema of the legs had completely subsided. There was a small right pleural effusion, but the left one had resolved completely. He was afebrile and the pulse was $80-100$. 
His general condition was good: increase of weight; normal blood sedimentation rate and blood count; and repeated examinations of the pleural fluid showed no change in cytology. Examination of pericardial fluid now showed 88 leucocytes per c.mm., now mainly lymphocytic.

The interesting features of the case at this stage were focused on the relatively good, temporary, response to treatment; the original difference in the cytology of the pleural and pericardial effusions; and the question whether the introduction of a pneumopericardium could have any influence in the prevention of chronic adhesive pericarditis (Pick's disease).

The patient spent twelve months in a sanatorium during 1943 and returned to the Leatherhead Hospital in 1944. His progress had been well maintained, but by April he was beginning to develop some engorgement of the neck veins and suggestive evidence of incipient constrictive pericarditis, confirmed by the development of a generalized anasarca with gross œdema of the legs, sacral œdema, ascites, and liver enlargement. He also had a high venous pressure and the circulation time (solution of saccharine, arm to tongue) was 28 seconds.

The possibility of a pericardial resection was under consideration, but in June, owing to the war situation, we were obliged to evacuate civilian patients to the North, where treatment was continued on the lines outlined, pericardial puncture yielding no fluid. He died suddenly in February 1945, and, most unfortunately, permission for post-mortem examination was not obtained. The case appears to have ended in a Pick's disease, secondary to a polyserositis.

\section{Case 5.}

A clerk, aged 31, came into Leatherhead Hospital complaining of breathlessness and swelling of the legs and feet. His disability dated back to a cold in 1939, followed by pneumonia, after which

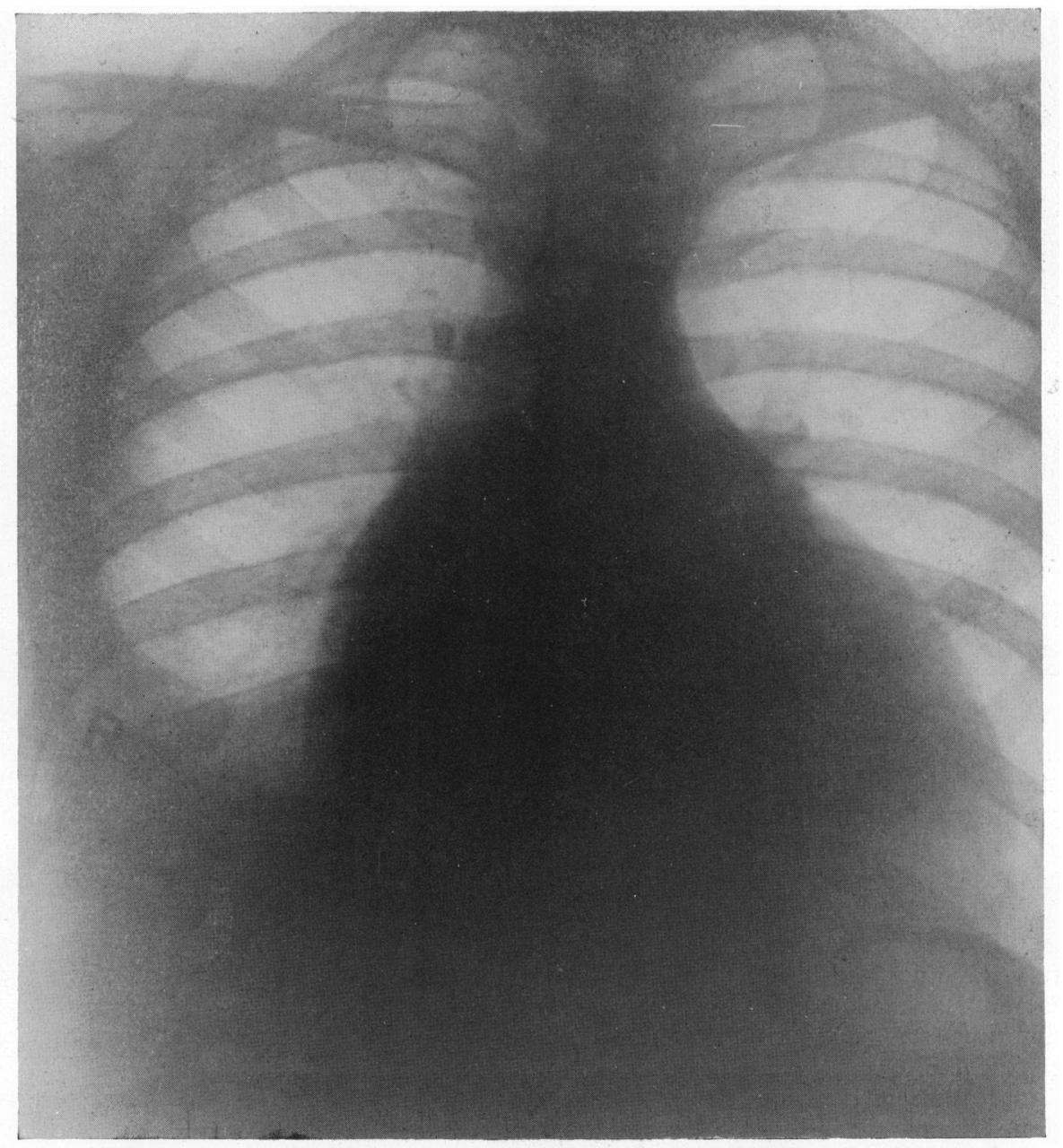

FIG. 8.-Case 5. Characteristic cardiac silhouette of a pericardial effusion with a right pleural effusion: pear-shaped, in the erect position: compare Fig. 9. 
he developed a right-sided pleural effusion with the symptoms mentioned. During convalescence the dyspnœa was a constant feature while the œdema was transient. Despite this he had been able to continue with his clerical job. He complained of pain in the form of a "tight constriction" below the ribs on exertion.

On admission he was dyspnœic, even at rest, and had a dry cough causing extreme breathlessness; he was plethoric and generally cyanosed, especially the arms and extremities; no clubbing of the fingers; no history of any acute rheumatism, but a suspicious family history of tuberculosis.

Examination. Neck veins engorged; heart grossly enlarged; apex beat palpable in fifth space, just outside normal limits; increased dullness to right and left; B.P. 120/90; trachea centrally placed; no significant murmurs.

X-ray examination. Cardiac silhouette, gross enlargement to right and left, highly suggestive of a pericardial effusion, pear-shaped in erect position (Fig. 8), but more rectangular in recumbent position (Fig. 9). A noteworthy feature, and of some diagnostic significance, was the marked

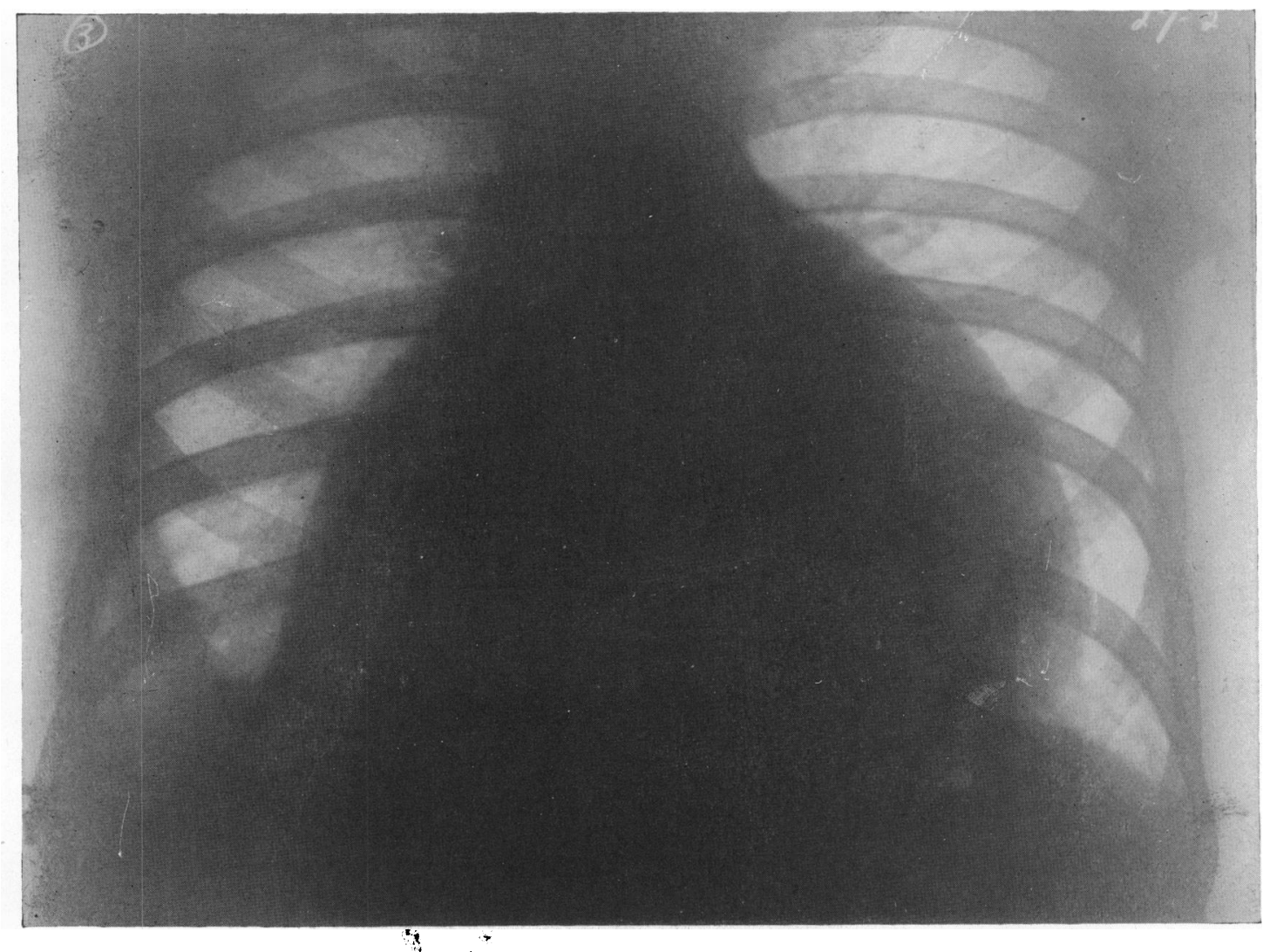

FIG. 9.-Case 5. The same patient recumbent, showing a cardiac silhouette more rectangular in shape.

calcification of the superior tracheo-bronchial glands, specially well seen in the lateral and right anterior oblique positions. Radioscopy showed almost complete absence of pulsation in the lower two-thirds of the right and left borders, but the upper third appeared to pulsate normally.

There was a right-sided pleural effusion which on puncture was straw-coloured, clotted readily on standing, revealed no organisms; sterile on culture; fluid mainly lymphocytic; protein content 4 per cent; blood count showed an erythrocytosis; blood sedimentation rate normal; pericardial puncture yielded no fluid but feel of needle gave impression of an obviously thickened pericardium with, possibly, a loculated or encapsulated effusion. Venous pressure $18 \mathrm{~cm}$. (sodium citrate); circulation time (arm to mouth) 37 seconds (normal 16). Electrocardiogram not pathological.

Liver palpable, $10 \mathrm{~cm}$. below the subcostal margin; gross œdema of legs.

My impression of this case has been that there has been a polyserositis, almost certainly of tuberculous origin-the calcified mediastinal glands seen radiologically give strong confirmatory evidence of this view-and he is now developing a constrictive pericarditis with gross thickening of the pericardium. Fluid may be present, in which case it is either loculated or is so thick that it cannot be aspirated through an ordinary needle. Gross cardiac enlargement (certainly not due to rheumatic 
heart disease), complete absence of cardiac murmurs, absence of a rheumatic history, low blood and pulse pressure, absence of cardiac pulsation on radioscopy, high venous pressure and prolonged circulation time, all strongly favour the diagnosis. In view of the problem in diagnosis Dr. Terence East kindly saw this case with me and agreed that, despite the normal cardiogram, the various findings strongly favoured this diagnosis. The patient was to see a thoracic surgeon with a view to a pericardial resection. Unfortunately he is schizophrenic and his confused mental state will not permit this for the present.

\section{Case 6 (Dr. John Parkinson).}

Whilst discussing the subject of tuberculous pericarditis with Dr. John Parkinson he brought to my notice a case he had under his care during the last war which presents a number of features of such interest that he has very kindly permitted me to quote extracts from it.

The patient, aged 22, was admitted to hospital at Rouen in 1917, ten days after complaining of a "cold", with weakness, cough, and loss of appetite. Previous history was good with no acute rheumatism, chorea, or tonsillitis.

Examination. A fairly well-nourished youth; whole face slightly puffy and glossy, eyelids not more than the rest; cheeks highly coloured, showing, with the lips, a degree of cyanosis. He could lie quite flat in bed but even when supported by two pillows there was slight objective dyspnœa. Only complaints were occasional cough and slight præcordial pain; temperature on admission 100; respirations 34; radial pulse very small, rate being approximately 130 and very irregular.

Examination of the heart showed no præcordial pulsation; area of deep cardiac dullness greatly increased, especially to left where it reached anterior axillary line; second left intercostal space dull to percussion; no abnormal dullness to right. On auscultation: no pericardial rub; heart sounds distant and unaccompanied by murmurs; dullness at base of left lung and in infra-axillary region, with crepitations and diminished breath sounds; bronchial breathing not heard. No abnormal signs discovered in abdomen and no pitting of legs on pressure.

Urine: examined three times, on each occasion a cloud of albumin present on boiling, but no casts found.

Radiographic examination showed pericardial sac greatly distended with fluid and a free left pleura.

Puncture of pericardium: performed five days after admission; 150 c.c. fluid aspirated (clear, except for a few small flakes), the needle showing the movements of the heart as it beat against it. Fluid sterile on culture, despite subsequent positive findings of tuberculosis. Aspiration produced little or no improvement; a course of sodium salicylate likewise unsuccessful.

For two months little change occurred. The patient ate and slept well; temperature irregularly remittent, at time intermittent, reaching 100 to 103 each evening; pulse 120-140 with extra systoles.

The pericardium was punctured on two further occasions; on the first 375 c.c. of clear fluid were removed; on the second 150 c.c. of slightly turbid fluid, but on both occasions it was sterile. During the last two months of his life there was moderate œdema of loins and legs. During the third month of the illness the general condition became worse. The sputum, previously scanty, now became profuse and purulent. When examined a fortnight before death it was found to contain tubercle bacilli. Apart from the signs at the left base nothing abnormal was found on examination of the lungs until the middle of April, when a few rhonchi were heard generally. By this time the patient was mentally dull during the day and mildly delirious at night. He became unconscious and died in May.

Post-mortem examination. This showed, briefly, a pericardial cavity occupying two-thirds of the space of the left pleural cavity and containing 2000 c.c. of dark straw-coloured fluid. The walls of the pericardial cavity were thickened, at some points to the extent of $0.5 \mathrm{~cm}$.; the surface of the heart was covered with thick fibrinous exudate (bread and butter pericardium). The heart was of moderate size with no valvular lesions, and the lung substance showed, fundamentally, miliary tuberculosis.

\section{Summary AND CONCLÚsions}

Tuberculous pericardial effusions, usually secondary to mediastinal gland tuberculosis, are probably not as uncommon as we have been led to believe. The condition may occur at all ages, and is not necessarily associated with gross symptomatology.

The combination of clinical, radiological, and cardiographic examinations and pathological investigations of the pericardial fluid are of great value in the diagnosis of the disease in the living subject.

Pericardial paracentesis in a suspected case is always advisable for diagnostic purposes and 
carries with it no undue risk. A characteristic straw-coloured lymphocytic exudate, as with a pleural effusion, should, despite negative findings for tubercle bacilli and guinea-pig inoculation, arouse very real suspicion of a tuberculous ætiology.

The induction of a pneumopericardium may have some therapeutic value, apart from its diagnostic value in assessing the size of the heart and the degree of thickening of the pericardium.

The relationship of a tuberculous pericardial effusion and polyserositis to a developing constrictive pericarditis (whose ætiology is probably tuberculous) is noted, and the great value of surgery in the quiescent stage of the latter must be appreciated.

The prognosis of tuberculous pericardial effusions, whilst dependent upon the nature of the fluid and the accompanying complications, is not necessarily associated with a high mortality rate. A fluid negative both on culture and to guinea-pig inoculation may occur and yet other diagnostic criteria may point to a tuberculous ætiology. In such cases the prognosis would appear to be correspondingly more favourable.

I am indebted to Dr. Terence East for the electrocardiograms of all my cases; to Dr. David Nabarro and Dr. John Dacie for pathological investigations; to Dr. C. Gray for biochemical investigations and to Dr. J. N. Cummings for photographs of Fig. 5 and 6.

\section{REFERENCES}

Barrett, A. M. and Cole, L. (1944). Brit. Heart J., 6, 4.

Beck, C. S. (1935). J. Amer. med. Ass., 104, 71.

Gloyne, S. R. (1944). Personal communication.

Hanneson, H. (1941). Tubercle, $20,79$.

Harvey, A. M. and Whitehill, M. R. (1937). Medicine, Baltimore, 16, 45-94.

Kornblum, K., Bellet, S., and Ostrum, T. M. (1933). Amer. J. Roentgen., 29, 203.

Norris, G. W. (1911). Cardiac Pathology, W. B. Saunders \& Co., Philadelphia.

Osler, W. (1893). Amer.J. med. Sci.,122,6.

Pagel, W. (1944). Personal communication.

Parkinson, J. (1944). Personal communication.

Pick, F. (1896). Z. klin. Med., 29, 385.

Roesler, H. (1943). Clinical Roentgenology of the Cardiovascular System. Thomas Springfield, Illinois, 2nd edition.

Sellors, T. H. (1944). Hunterian lecture. Royal College of Surgeons.

Suzman, S. (1943). Brit. Heart J., 5, 1 .

White, Paul (1935). Lancet, 2, 539. 